\title{
Simulated Avalanche Formation Around Streamers in an Overvolted Air Gap
}

\author{
Chao Li, Ute Ebert, and Willem Hundsdorfer
}

\begin{abstract}
We simulate streamers in air at standard temperature and pressure in a short overvolted gap. The simulation is performed with a 3-D hybrid model that traces the single electrons and photons in the low-density region while modeling the streamer interior as a fluid. The photons are followed by a Monte Carlo procedure, just like the electrons. First simulation results are presented here.
\end{abstract}

Index Terms-Gas discharge, hybrid model, photoionization, streamers.

$\mathbf{P}$ HOTOIONIZATION is recognized as an important ingredient for the propagation of positive streamers in air (but not necessarily in other gases) [1]. In negative streamers, velocity and diameter seem to be modified by photoionization only in sufficiently high background fields according to fluid models [2], [3]. In this work, photoionization is included in our hybrid model [5], [6] on the particle level as implemented in [4]. This means that single electrons and photons are followed as particles in the low-density region to describe the potential runaway of electrons as well as the density fluctuations arising from the discreteness of the individual particles.

The photoionization is implemented in two steps: First, ionizing photons are emitted, and then, the photons are absorbed in an ionization event.

1) The emission rate of ionizing photons is assumed to be proportional to the number of ionizing electron-neutral collisions. The hybrid model couples a particle model with a fluid model in space, as discussed in [5] and [6]. In both the particle region and the fluid region, photons are generated by a Monte Carlo process based on the local rate of ionizing electron-neutral collisions.

2) The frequencies of ionizing photons are assumed to be uniformly distributed within $2.925-3.059 \mathrm{PHz}$. The mean free path length of photons is a function of the frequency as given in [7]. The flight distance of the photons is then sampled from their mean free path length as for the electrons in [5] and [6]. Another random number determines the flight angle of photons under the assumption of isotropic emission; distance and angle determine the

Manuscript received December 1, 2010; revised March 20, 2011; accepted March 27, 2011. Date of publication August 30, 2011; date of current version November 9, 2011. This work was supported in part by Project EWR Science of EUV 10010305 and in part by STW Project 10118.

C. Li is with the Department of Applied Physics, Eindhoven University of Technology, 5600 MB Eindhoven, The Netherlands.

U. Ebert and W. Hundsdorfer are with Centrum Wiskunde \& Informatica, 1098 XG Amsterdam, The Netherlands.

Digital Object Identifier 10.1109/TPS.2011.2163528 location of absorption. When absorption occurs in the particle region, a new electron-ion pair is created. When absorption occurs in the fluid region, the local particle densities change correspondingly.

The simulation results are shown in Fig. 1. Two planar electrodes at a distance of $1.17 \mathrm{~mm}$ create a background field of $-100 \mathrm{kV} / \mathrm{cm}$ in air at standard temperature and pressure. The initial condition consists of a Gaussian distribution of 500 electron-ion pairs near the cathode. The simulation is carried out on a grid of $256 \times 256 \times 512$ points with cell length $\Delta x=$ $\Delta y=\Delta z=2.3 \mu \mathrm{m}$ with Neumann boundary conditions in the lateral direction. The time step is $\Delta t=0.3 \mathrm{ps}$. Fig. 1 shows the bulk of electrons with a density $>10^{13} / \mathrm{cm}^{3}$ (left), the charge density (middle), and the strength of the electric field in the $z$-direction $E_{z}$ (right) at four different times. At $t=0.375 \mathrm{~ns}$, a double-headed streamer starts to form. At $t=0.4875 \mathrm{~ns}$, an avalanche exceeds the density threshold and appears in the electron bulk plot. At $t=0.6 \mathrm{~ns}$, an avalanche (at the right of the primary streamer) propagates across the $y=0$ plane and therefore can be seen in all three plots. At $t=0.7125 \mathrm{~ns}$, the space charge layer of the primary streamer, as well as the electric field, is strongly perturbed. Most photons are absorbed within a few micrometers, but with a small probability, photons travel further and ionize molecules at a rather long distance, and the electrons liberated by these far-traveled photons initiate new avalanches.

In conclusion, by introducing a particle description of the photoionization in our hybrid model, we are able to study the density fluctuations induced by single photons.

\section{REFERENCES}

[1] G. Wormeester, S. Pancheshnyi, A. Luque, S. Nijdam, and U. Ebert, "Probing photo-ionization: Simulations of positive streamers in varying $\mathrm{N}_{2}: \mathrm{O}_{2}$ mixtures," J. Phys. D, Appl. Phys., vol. 43, no. 50, Dec. 2010, 505201.

[2] A. Luque, U. Ebert, C. Montijn, and W. Hundsdorfer, "Photoionisation in negative streamers: Fast computations and two propagation modes," Appl. Phys. Lett., vol. 90, no. 8, Feb. 2007, 081501.

[3] A. Luque, V. Ratushnaya, and U. Ebert, "Positive and negative streamers in ambient air: Modeling evolution and velocities," J. Phys. D, Appl. Phys., vol. 41, no. 23, Dec. 2008, 234005.

[4] O. Chanrion and T. Neubert, "A PIC-MCC code for simulation of streamer propagation in air," J. Comput. Phys., vol. 227, no. 15, pp. 7222-7245, Jul. 2008.

[5] C. Li, U. Ebert, and W. Hundsdorfer, "Spatially hybrid computations for streamer discharges with generic features of pulled fronts: I. Planar fronts," J. Comput. Phys., vol. 229, no. 1, pp. 200-220, Jan. 2010.

[6] C. Li, U. Ebert, and W. Hundsdorfer, "3D hybrid computations for streamer discharges and production of run-away electrons," J. Phys. D, Appl. Phys., vol. 42, no. 20, Oct. 2009, 202003.

[7] M. B. Zheleznyak, A. Kh. Mnatsakanyan, and S. V. Sizykh, "Photoionization of nitrogen and oxygen mixtures by radiation from a gas discharge," Teplofizika Vysokikh Temp., vol. 20, no. 3, pp. 423-428, May/Jun. 1982. 

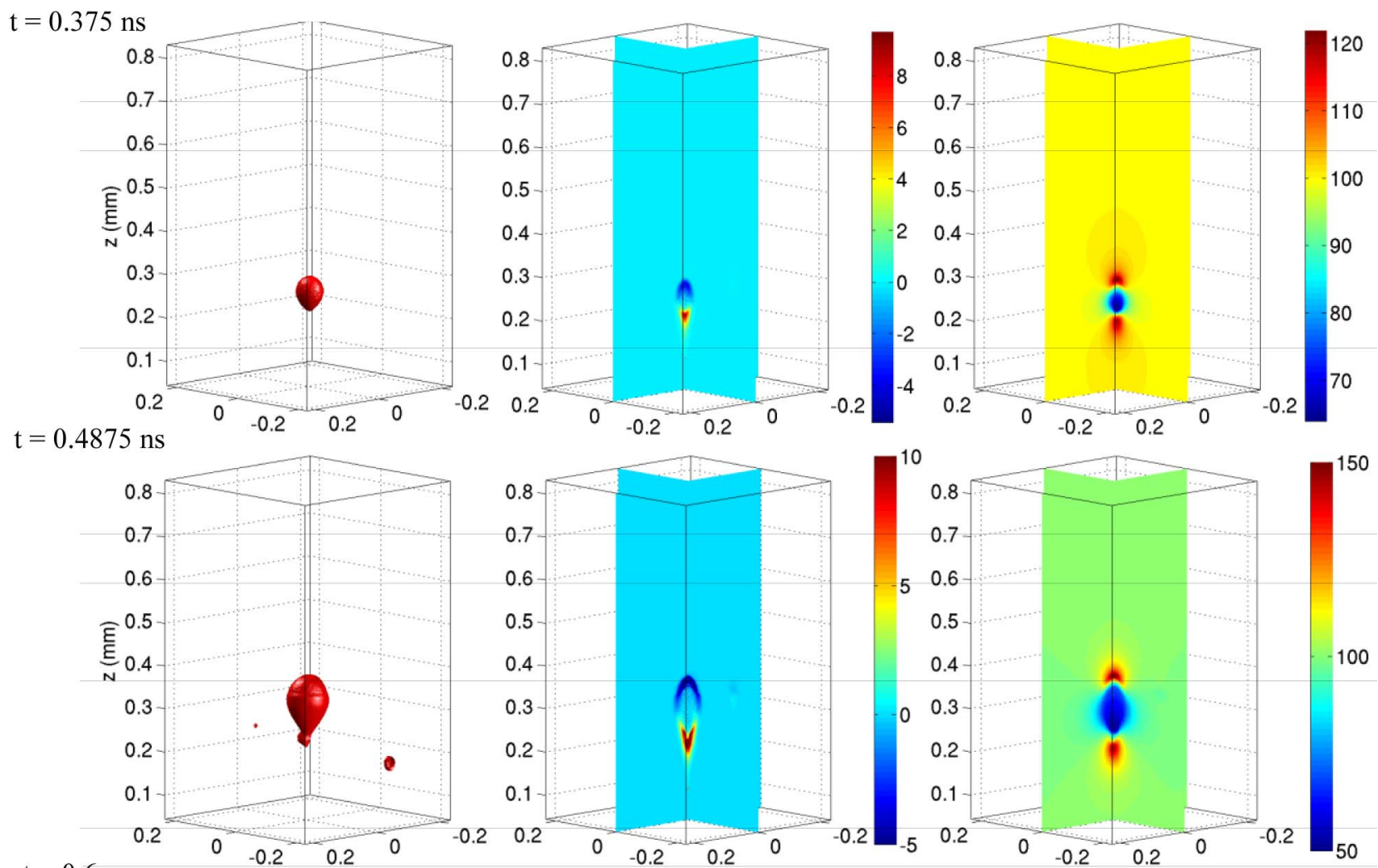

$\mathrm{t}=0.6 \mathrm{~ns}$
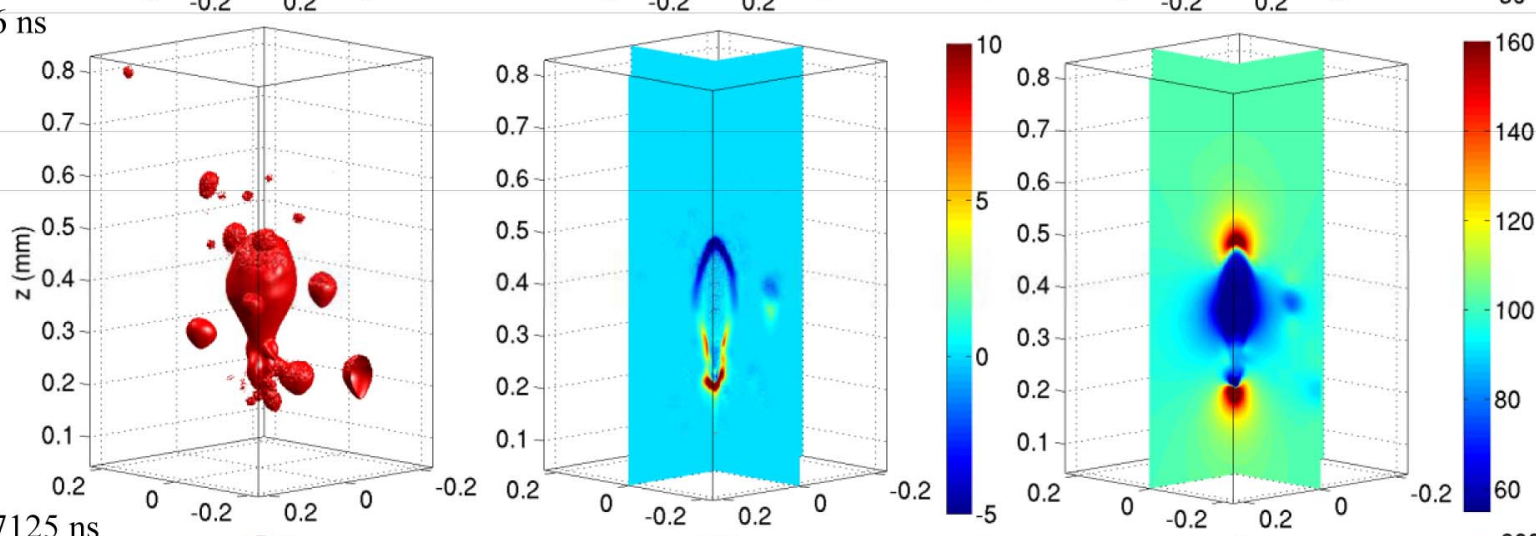

$\mathrm{t}=0.7125 \mathrm{~ns}$

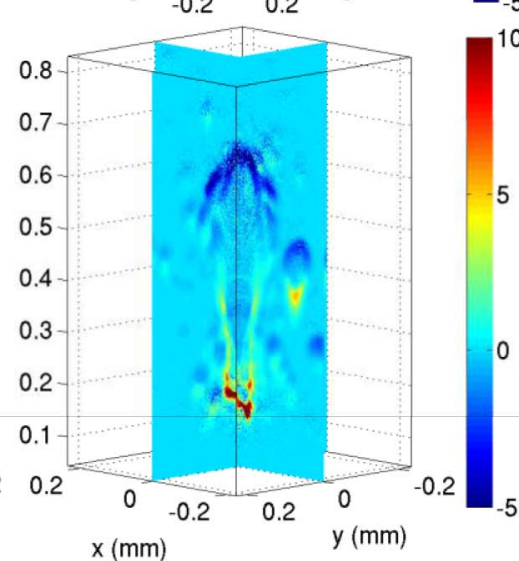

Charge Density (in $10^{-6} \mathrm{C} / \mathrm{cm}^{3}$ )

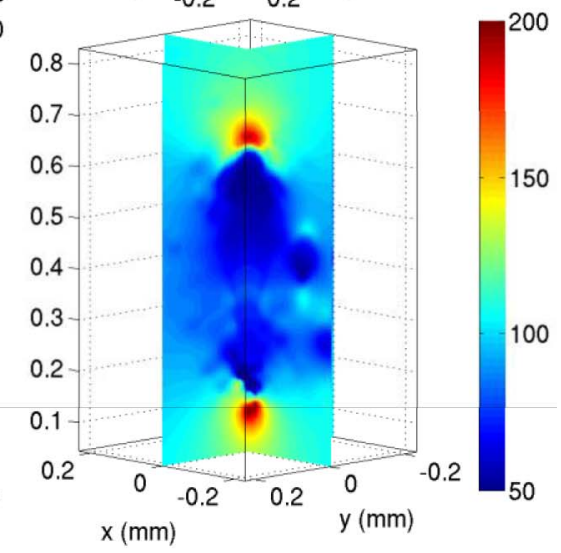

$\mathrm{E}_{\mathrm{z}}($ in $\mathrm{kV} / \mathrm{cm})$

Electrons

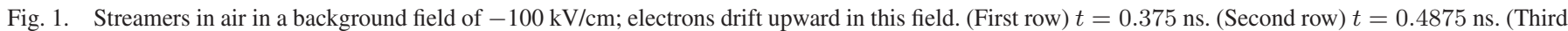
row) $t=0.6 \mathrm{~ns}$. (Fourth row) $t=0.7125 \mathrm{~ns}$. The columns from left to right are the surfaces where the electron density exceeds $10^{13} / \mathrm{cm}^{3}$, the charge density (in $10^{-6} \mathrm{C} / \mathrm{cm}^{3}$ ), and the electric field $E_{z}($ in $\mathrm{kV} / \mathrm{cm})$. 